\title{
Occlusion as a depth cue in the Wheatstone-Panum limiting case
}

\author{
HIROSHI ONO, KOICHI SHIMONO, and KOICHI SHIBUTA \\ York University, North York, Ontario, Canada
}

\begin{abstract}
We examined the hypothesis (Ono \& Wade, 1985) that occlusion of far stimuli by a near one on the same visual line can operate as a depth cue in stereograms containing different numbers of targets in the two eyes. By controlling eye positions, we created conditions in which the visual system could interpret the retinal images as originating from stimuli on the visual axis of one eye and also created other conditions in which the origin of the retinal images was ambiguous. In Experiment 1, we presented two lines to one eye and a single line to the other eye. When the image of the line on the temporal side of the line pair on one retina fused with the image of the single line on the other retina, the nonfused line appeared farther away more often than it did when the image on the nasal side fused. In Experiment 2, we used two differently shaped stimuli. In the condition in which the nonfused stimulus represented an object being occluded, it appeared farther away more often than in the four conditions in which it did not. In Experiment 3, we extended the idea to three different objects. When the middle of the three images fused with the single image, the nonfused stimulus appeared farther when it could be interpreted as being occluded than when it could not. In the condition in which the most temporal image fused with the single image, the nonfused stimuli appeared farther than in the condition in which the most nasal one fused. The results supported the hypothesis that occlusion plays a role in depth perception in the Wheatstone-Panum limiting case.
\end{abstract}

Wheatstone (1838) and Panum (1858/1940) demonstrated that a stereogram which presents one stimulus to one eye and two stimuli to the other produces a threedimensional percept. This stimulus is usually known as the Panum limiting case, but in this paper it will be referred to as the Wheatstone-Panum limiting case to honor Wheatstone for demonstrating the phenomenon first (Ogle, 1962; Ono \& Wade, 1985; Westheimer, 1976). Despite this case's early discovery and a mild but continued interest in it (see, e.g., Brewster, 1844; Kaufman, 1976; Linschoten, 1956; Marr, 1982; Westheimer, 1986), the depth seen is still not understood well. In some experiments (e.g., Brewster, 1844), depth has not been seen. In other experiments (Gettys \& Harker, 1967; Ono \& Wade, 1985), the direction of the perceived depth has not been consistent across observers and across conditions. Some of this variability may be explained by a recent hypothesis (Ono \& Wade, 1985) examined in this study.

The hypothesis asserts that the Wheatstone-Panum limiting case represents two objects on a visual line of

This research was supported by Grant A0296 from the Natural Sciences and Engineering Research Council of Canada and by a grant from the Institute for Space and Terrestrial Science. The results of Experiments 1 and 2 described in this paper were reported by Shimono and Ono (1986) at the Association for Research in Vision and Ophthalmology meeting in Sarasota, FL. The authors wish to thank C. Dengis, J. Finley, E. Gonzalez, L. Gunther, I. Howard, R. Korn, A. Mapp, K. Phillips, M. Steinbach, and $N$. Wade for helpful comments on an earlier version of this paper and M. Ohmi for his help in preparing the apparatus for Experiment 3. Correspondence should be addressed to Hiroshi Ono, Department of Psychology, York University, North York, Ontario, Canada M3J 1P3. one eye, and that the perceived depth results from its interpretation as a near object occluding another object farther away from that eye. To note that the stereogram for the limiting case represents two objects on a visual line is not new (see, e.g., Arditi, 1986; Brewster, 1844; Julesz, 1971; Kaufman, 1974; Shimono \& Ono, 1986); what is new is the explicit assertion that the visual system uses occlusion as a cue to depth. The occlusion cue. in the limiting case differs from the traditionally defined cue, sometimes called interposition, in two ways. The traditional cue is monocular; in the limiting case, however, the information about the depth is available only binocularly. That is, one stimulus to one eye or the two stimuli to the other eye considered alone do not provide information about depth. Moreover, the monocular cue provides only ordinal or qualitative depth information, whereas in the limiting case there is information about the magnitude of depth. That is, as the distance becomes larger between the two objects on a visual line of one eye, the separation between the two retinal images in the other eye also becomes larger. This geometric analysis shows that the limiting case is analogous to those recently examined by Nakayama and Shimojo (1990) and Shimojo, Silverman, and Nakayama (1988), who found that the binocularly defined occlusion was an effective cue and that the depth covaried with the retinal separation.

The hypothesis that occlusion can be involved in the Wheatstone-Panum limiting case suggests that not all stimulus conditions that meet the description of the limiting case provide such a cue. To understand the stimulus conditions in which occlusion may or may not operate 
A. Tworobjects along the visual line of the right eye

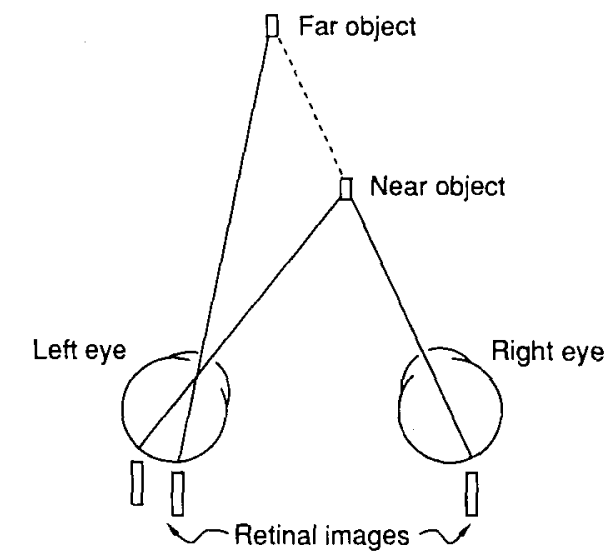

B. An example of stereograms for the Wheatstone-Panum limiting case

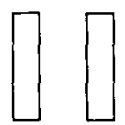

Left eye's view

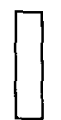

Right eye's view
Figure 1. Two stimuli along a visual line of the right eye and their retinal images (A) and the stereogram representing them (B). as a depth cue, consider two objects on a visual line of one eye as in Figure 1A, which shows two bars arranged so that the near one occludes the far one from one eye. Note that the stimulus arrangement produces only one image in one eye while it produces two images in the other, like those formed by the usual stereogram for the Wheatstone-Panum limiting case (see Figure 1B). According to the hypothesis, depth is seen when the visual system interprets: (1) the image on the temporal side of the pair of targets on one retina and the single image on the other retina as arising from the near object, and (2) the image on the nasal side of the pair of targets as arising from the far object occluded from one eye by the near object, as is depicted in Figure 2A. However, if the visual system interprets the single image on one retina and the image on the nasal side of the other retina as arising from the same object (shown in Figure 2B), the depth becomes ambiguous in this condition. In this case, the image on the temporal side has no partner, and the hypothesis makes no predictions regarding the relative position of the two perceived objects. (Throughout this paper, the terms temporal and nasal are used to connote "more temporal and more nasal on the retina" or "templeward and noseward," not necessarily "temporal hemiretinal and nasal hemiretinal." Moreover, the terms will be restricted to describe the retinal location rather than the visual field location.)

The hypothesis was examined in three experiments. Each had conditions in which occlusion was likely to operate as a depth cue and conditions in which this was less

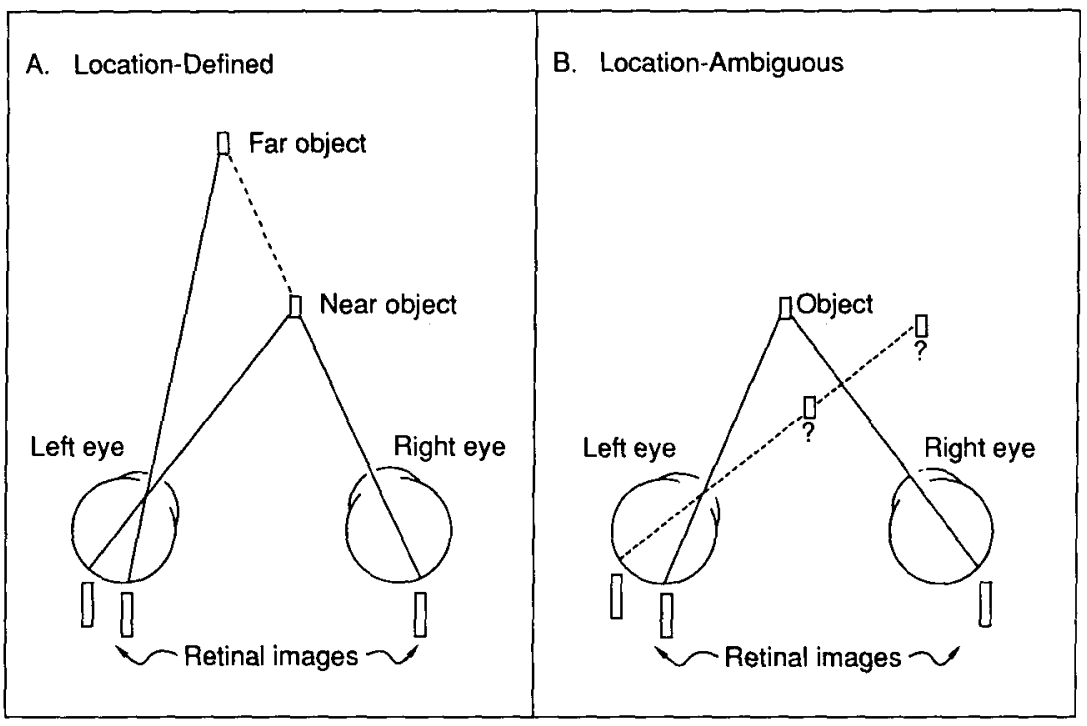

Figure 2. Difference between the location-defined condition $(A)$ and the location-ambiguous condition (B) in Experiment 1. Note the uncertain distal location for one of the retinal images in the left eye (B). 
likely. To produce such conditions, we manipulated the retinal locations of the stimuli in Experiments 1 and 3; the shape and the size of the stimuli, in addition to their retinal locations, were manipulated in Experiment 2. To determine the robustness of the depth seen with this kind of stimulus, we produced apparent depths with retinal disparity as comparisons in Experiment 3. In all three experiments, the primary aim was to examine the viability of the occlusion hypothesis; it was not to eliminate the other hypotheses that might be used to explain the depth seen in the Wheatstone-Panum limiting case. A discussion of the different hypotheses occurs in the last section of this paper.

\section{EXPERIMENT 1}

Controlling the eye position brought either the nasal or the temporal image on one retina into binocular correspondence with the single image on the other retina. In this way, we manipulated the likelihood that occlusion would operate as a cue. Occlusion is more likely to operate when the temporal image on one retina and the single image on the other retina are in binocular correspondence. We expected that these two images would fuse as the near object and would appear in the fixation plane, while the image on the nasal side would appear as the far object occluded by that in the fixation plane (see Figure 2A). In contrast, when the image on the nasal side of one retina and the single image on the other retina are in correspondence, there is no reasonable interpretation for the origin of the image on the temporal side, either in terms of occlusion or of any other geometric arrangement. Again, we expected the two images on corresponding points to appear as an object in the fixation plane; but, if the two images are interpreted as arising from the same object, there is no good interpretation for the origin of the temporal image. That is, the object that provides the retinal image on the temporal side of one retina should also provide a retinal image to the other eye; but there is no such image, and thus the location of the perceived object with the temporal image is ambiguous (see Figure 2B). In Experiment 1, two experimental conditions fitted these descriptions; the first was called the locationdefined condition and the second, the location-ambiguous condition. For the control condition, we presented stereograms that produced two images in each eye without retinal disparity.

\footnotetext{
Method

Stimuli and Apparatus. A Grinell Graphic System controlled by an LSI-11/23 computer generated the stimuli on a Hitachi HM2713 monitor. The screen was located $1 \mathrm{~m}$ from the observer's corneal plane. Polarized filters allowed the right eye to see the left half of the screen and the left eye to see the right half. The convergence distance required to fuse the two halves of the screen was about $40 \mathrm{~cm}$. A -1.5 diopter lens placed in front of each eye matched the required accommodation to this convergence distance, and a variable diopter prism also in front of each eye allowed adjustments of the location of retinal images.
}

Five stereograms were used: two for the location-defined condition, two for the location-ambiguous condition, and one for the control condition. In each set, a nonius stimulus was presented to provide a fixation target. In the two experimental conditions, the stereograms were like those shown in Figure 1B. One field had one vertical line (or bar), and the other field had two vertical lines. The two sets of stereograms differed in the relative location of the lines and nonius stimulus. Each set had two stereograms to counterbalance each field of the stereogram's being on the right or left side. In the control condition, the stereogram differed from the four experimental stereograms. Two fields had two vertical lines so that the nasal image of the line in one eye fell on the corresponding points of the temporal image of the line in the other eye. The lines used in each stereogram were $58^{\prime}$ of arc high and $5^{\prime}$ of arc wide. The horizontal separation of the two lines was $30^{\prime}$ of arc. The luminance of each line was $5.8 \mathrm{~cd} / \mathrm{m}^{2}$.

The nonius stimulus was located above the lines just described and consisted of two vertical lines $\left(7^{\prime} \times 16^{\prime}\right.$ of arc) and two horizontal lines $\left(37^{\prime} \times 6^{\prime}\right.$ of arc). One vertical line and one horizontal line presented to each eye formed a $\mathrm{T}$ in one eye and an upside down $T$ in the other. See Figure 3 for an example. The junction of the two lines of the $T$ was $68^{\prime}$ of arc above the top of the lines. When the composite nonius stimulus was fixated, the fixation or convergence response was judged appropriate for the experiment if the two vertical lines of the nonius stimulus appeared to be collinear, giving the appearance of a cross or a plus sign. That is, one of the two lines in one eye and the single image on the other retina fell on corresponding points for the experimental conditions. If they were apparently not collinear, the variable diopter prism in front of each eye was adjusted until they were.

Procedure. Observers were instructed to fixate the center of the nonius stimulus and to push a button on the control box to begin a trial. They were asked to push the button only when the nonius lines were collinear. A stereogram was presented for $100 \mathrm{msec}$ to minimize the chance of eye movements during the stimulus presentation. The task was to report whether or not the two perceived lines appeared in the same plane, and if they were not, which line appeared closer.

Each observer had five blocks of trials. Each block consisted of 20 trials in which the five different stereograms were presented four times each in random order. Between each block, the observer rested for as long as he or she wanted.

Observers. Eleven members of the university community $-6 \mathrm{fe}-$ males and 5 males-participated. They ranged in age from 23 to 36 years, and they reported having normal or corrected-to-normal acuity and stereopsis.

\section{Results and Discussion}

We first examined the response in each trial to determine whether depth was reported. Then, the depth responses in the two experimental conditions were coded according to whether the nonfused line was reported to be behind or in front of the fused line. (The fused line is one of the two perceived lines; it is on the left side [right], when the left [right] line of the two presented to one eye projects to the corresponding point of the single line presented to the other eye. The term fused is used here for a descriptive purpose. The question of whether two lines are seen because one of the three retinal images is suppressed is not addressed in this paper.) The frequencies of "front," "behind," and "same-depth" responses were converted into percentages for each observer. In the control condition, however, only the percentages of "depth" and "no depth" were computed. An arcsine 


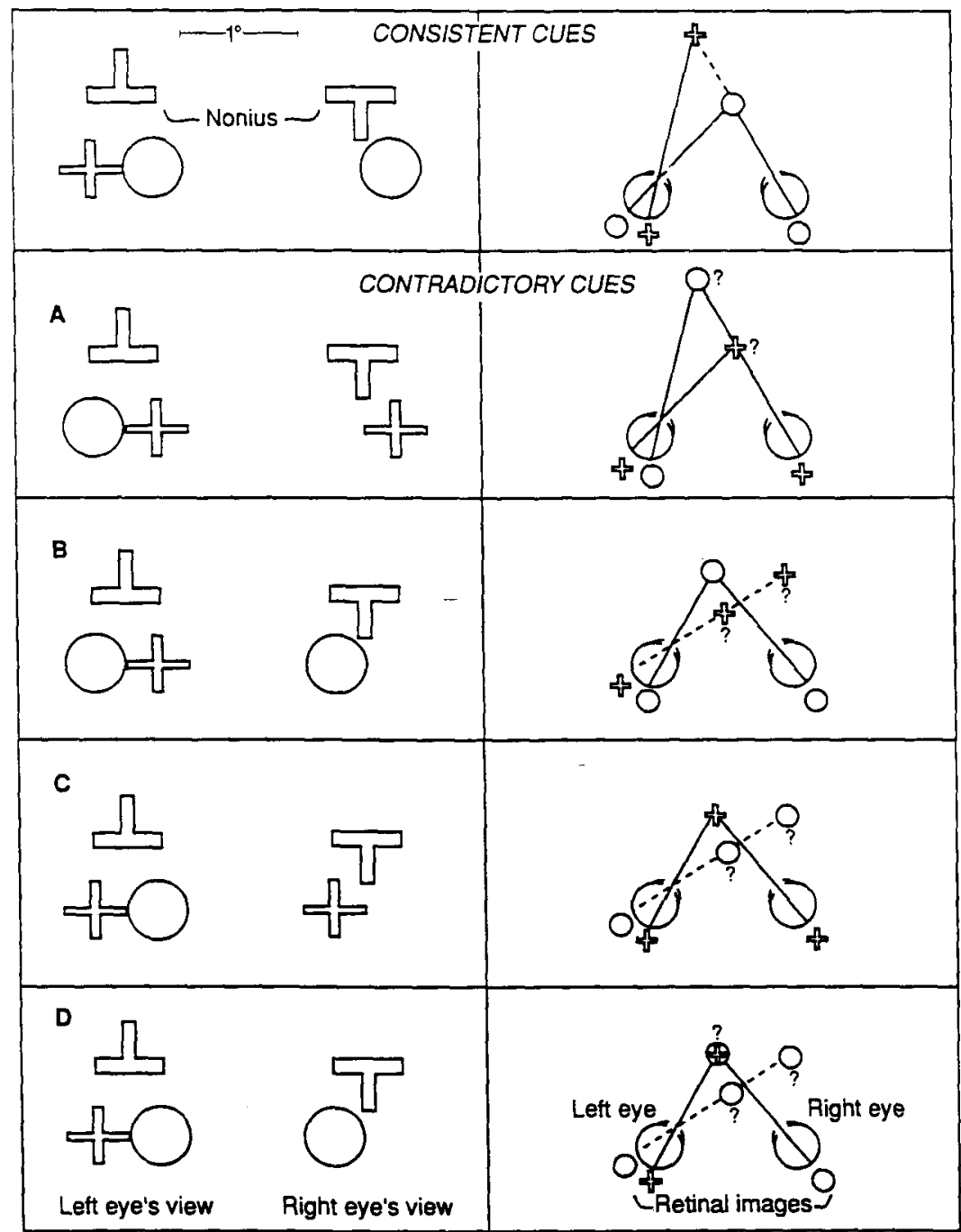

Figure 3. Stereograms used in Experiment 2, and the possible distal locations for the retinal images produced by the stereogram. Although the stimuli are depicted with contours, they actually consisted of luminous patterns.

transformation was performed on each percentage to homogenize the variances (Walker \& Lev, 1953), and the transformed value was used as a basic unit of analyses to follow. Table 1 shows the means of the values across observers. The values shown are percentages transformed back from the mean of the arcsine values.

Our stereograms for the Wheatstone-Panum limiting case were effective in producing three-dimensional perception. The experimental stereograms produced more depth perception and fewer "same-depth" responses than did the control stereograms with zero disparity. Between the location-defined and control conditions, the difference in the percentages of "same-depth" responses was 74.5\% $[t(10)=10.31, p<.001]$, and between the control and the location-ambiguous conditions, the difference was $61.9 \%[t(10)=6.98, p<.001]$. Because of these sta- tistical differences, we were able to test the occlusion hypothesis.

The main prediction of the hypothesis concerns the difference between the two experimental conditions. The mean percentage of "behind" responses was over $90 \%$ in the location-defined condition and was greater than that in the location-ambiguous condition. The difference in the percentages was $32.6 \%[t(10)=3.71, p<.01]$. This statistically significant difference supports the hypothesis.

Whether this conclusion is compatible with the results of other studies is difficult to say, because three relevant studies (cited in Kaufman, 1976; Kaufman \& Lane, 1979; cited in Ogle, 1962) have not been published as journal articles. Ogle (1962) reports that "the depth sensation frequently does not occur if the second line for one eye is exposed only momentarily"' (p. 376), but whether the reti- 
Table 1

Mean Percentages of Nonfused Stimulus Appearing Behind, in Front of, and in the Same Depth as the Fused Stimulus in Experiment 1

\begin{tabular}{|c|c|c|c|}
\hline \multirow[b]{2}{*}{ Conditions } & \multicolumn{3}{|c|}{ Responses } \\
\hline & "Behind" & "Front" & "Same Depth" \\
\hline Location defined & 92.7 & 3.4 & 3.6 \\
\hline Location ambiguous & 60.1 & 16.8 & 16.2 \\
\hline Control & \multicolumn{2}{|c|}{21.8} & 78.1 \\
\hline
\end{tabular}

nal stimulation corresponds to our location-ambiguous or location-defined condition is not clear. His Figure 16 suggests that it corresponds to the location-ambiguous condition. Kaufman (1976) reports that he was unable to achieve depth "when convergence was set with a nonius marker immediately prior to a 100 msec exposure to limiting case stereograms having disparities of $13.4 \mathrm{~min}$ of arc and 33.4 min of arc" (p. 228), but again it is not clear to which experimental condition of this study his condition corresponds. Kaufman and Lane (1979) report that the "uniocular spot was presented prior to the binocular spots" and on the basis of this procedure, it can be argued that occlusion information may be ineffective. Even if the convergence and retinal-image locations corresponded to those of the location-defined condition, without the "binocular spot" there is no information that the "uniocular spot" resulted from the occlusion. Shimojo et al. (1988) have demonstrated that the sequence of presenting binocular and monocular stimuli is critical in the operation of an occlusion cue. To summarize, in these three studies, depth was not seen, but the depth information may have been ambiguous.

Given this summary, the result in the location-ambiguous condition of this study warrants further discussion. In this condition, the nonfused line appeared reliably behind; the percentage of the "behind" responses was greater than that of the "front" responses by $43.3 \%[t(10)=2.31$, $p<.05$ ], and 9 out of 11 observers made the "behind" responses more frequently than the "front" responses (sign test, $p<.033$ ). This finding is interesting, because it does not conform to what Panum (1858/1940) found. He states:

Under all circumstances, that one of the double lines which is in reality closest to the single line [the right one of the left eye view in Figure 1B which produces the most leftward (temporal) retinal images in Figure 1A] seems farther forward than the other and it is immaterial whether the single line is made coincident with one or the other of the double lines. (p. 112)

To apply this rule to the stereogram shown in Figure 1B, what appears on the right side in the visual field should appear to be closer than what appears on the left, whether the retinal location of the single line of the right eye's view corresponds to the right one or the left one of the left eye's view. Panum states the rule in terms of the distal stimulus; in terms of two proximal (retinal) stimuli in one eye, the rule asserts that what stimulates the temporal side would appear closer than what stimulates the nasal side. The vio- lation of this empirical rule found in Experiment 1 will be discussed in relation to Experiment 2. For now, it is important to note that the mean percentage of responses in the location-ambiguous condition that agree with Panum's rule was only $16.8 \%$ (the percentage of "front" responses) in contrast to $92.7 \%$ in the location-defined condition. The difference between these two percentages is $75.9 \%$, which is considerably larger than the difference of $32.6 \%$ found between the two percentages of responses that the nonfused line appeared to be "behind." Accordingly, an analysis of the directions of depth in terms of agreement with Panum's empirical rule, rather than in terms of the nonfused line's appearing behind, indicated a greater statistical significance for the difference between the two experimental conditions $[t(10)=10.69, p<.001]$.

\section{EXPERIMENT 2}

We tested the occlusion hypothesis further with the idea that an occlusion interpretation should also operate with two differently shaped objects along a visual line of one eye. The retinal images of these objects would be different from those in the Wheatstone-Panum limiting case, but the effect on the depth perception should be the same, provided that the near object is large enough to occlude the far object. In Experiment 2, we presented stereograms like those shown on the left panels in Figure 3. The stereogram shown on the top left panel represents two objects along a visual axis, with a disk in front of a cross. The real-life counterpart is shown on the top right panel. With this stereogram, the underlying perceptual process that operates should be the same as that in the location-defined condition in Experiment 1, when the nonius criterion is met. The occlusion hypothesis predicts that the nonfused stimulus would appear behind the fused one. For the stereogram depicted on the top left side of Figure 3, the cross should appear behind the disk as depicted on the top right side of Figure 3. With the four stereograms on the bottom, however, the depth information is contradictory; thus, the locations of the two perceived objects are not defined. The occlusion hypothesis makes no prediction for these stereograms regarding direction of depth. See the four bottom right panels in Figure 3. The stereogram shown in panel A represents the cross in front of the elliptical disk, but the image of the cross is too small to occlude the disk. Thus the cues are contradictory, because the unoccluded disk has no corresponding contours in the right image. Those shown in panels B and C are similar to that in the locationambiguous condition in Experiment 1, but they differ in that the retinal images in Experiment 1 could have arisen from two objects with the "wrong" fixation (the intersection of two visual axes at the far object), whereas the retinal images produced by the stereograms in panels $B$ and $C$ could not. The cross in panel $B$ and the disk in panel $\mathrm{C}$ for the left eye each have no partner in the right eye. The stereogram shown in panel D is more like that in the location-ambiguous condition; the retinal images can arise from a real-life counterpart-namely, a disk (near) and a cross (far) along a visual line. If we were 
to disregard the eye position, this stereogram could be classified as belonging to the consistent-cue condition. However, because of the eye position imposed by the nonius stimulus, what is seen is a disk with a cross on it and an empty disk. There is no reasonable interpretation for the physical origins of what is seen, as was the case in the location-ambiguous condition in Experiment 1. In Experiment 2, the condition in which the stereogram on the top was presented was called the consistent-cue condition; the conditions in which other stereograms were presented were called contradictory-cue conditions.

\section{Method}

We presented 10 stereograms with the apparatus used in Experiment 1 . Figure 3 shows 5 of them. The other five were like those shown in the figure, except that the stimuli for the right eye were switched to those for the left eye and vice versa. The switch within the stereogram shown for the consistent-cue condition (the top of Figure 3) made the second stereogram for the contradictory-cue condition (panel B), and the switch within the stereogram in panel A made the second stereogram in panel $C$. The switch within the stereogram in panel $\mathrm{D}$, however, made another stereogram for that condition. (Two other stereograms can be made for this condition by placing the cross on the right side and the disk on the left side. They were not included, however, in order to shorten the experiment.) The horizontal angular separation between the center of the cross and the center of the disk was 33' of arc. The vertical angular separation between the center of the nonius stimulus and the top of the disk or the cross was $41^{\prime}$ of arc. The horizontal and vertical dimensions of the disk and the cross were $33^{\prime}$ of arc and 29' of arc, respectively. The width of the lines of the cross was $4^{\prime}$ of arc. The nonius stimulus was the same as the one used in Experiment 1 and is shown in Figure 3.

The procedure was identical to that of Experiment 1, except that each stereogram was presented 10 times. One hundred trials were run in five blocks for each observer. Each block had 20 trials in which 10 different stereograms were presented twice in random order. The observers were the 11 who participated in Experiment 1.

\section{Results and Discussion}

As in Experiment 1, we coded the "right," "left," or "same-depth" response in each trial for whether the nonfused stimulus on the noncorresponding points appeared behind, in front of, or at the same depth as the fused or composite one on corresponding points. The term composite is used to distinguish between what was seen with the stereogram in panel D (or with its companion stereogram) from what was seen with the rest of the stereograms. With the stereogram in panel $D$, observers saw a cross and a disk on the left side of the visual field and a plain disk on the right side. For the other stereograms, observers saw a disk on one side and a cross on the other. For each observer and each condition, we computed the percentages of the trials in which the nonfused stimulus appeared behind, in front, and at the same depth. As in Experiment 1, an arcsine transformation was performed on each percentage and the transformed value was used as the basic unit of analysis. The mean values in Table 2 are the percentages that are transformed back from the mean of the arcsine values.

To test the hypothesis, we performed a one-way repeated measures analysis of variance on the "behind"
Table 2

Mean Percentages of Nonfused Stimulus Appearing Behind, in Front of, and in the Same Depth as the Fused Stimulus in Experiment 2

\begin{tabular}{lccc}
\hline & \multicolumn{3}{c}{ Responses } \\
\cline { 3 - 4 } Conditions & "Behind" & "Front" & "Same Depth" \\
\hline Contradictory cues & & & \\
A & 42.1 & 25.1 & 27.4 \\
B & 46.7 & 16.3 & 30.7 \\
C & 17.4 & 39.4 & 36.8 \\
D & 14.5 & 58.6 & 22.5 \\
Consistent cues & 84.1 & 4.4 & 11.3 \\
\hline
\end{tabular}

data. The main effect was statistically significant $[F(4,40)$ $=12.55, p<.001]$. The post hoc analyses (Tukey test) showed that the mean in the consistent-cue condition was significantly higher than each of the four means in the contradictory-cue conditions $(p<.001)$ for the smallest difference-namely, the consistent-cue condition versus Condition B. [The analysis of the front data shows essentially the same effects, $F(4,40)=8.62, p<.001$.] These significant main effects and the significant differences strongly support the occlusion hypothesis.

To test whether the results in each of the contradictorycue conditions agreed or disagreed with Panum's empirical rule noted in Experiment 1, we examined the difference between the mean percentage of "behind" and of "front" responses. The direction of difference obtained in Condition B is in violation of Panum's rule as in the location-ambiguous condition in Experiment 1, because the stimulus projecting to the temporal side of the retina appears farther away more frequently. The difference, however, is not statistically significant. The direction of difference in Conditions A, C, and D is in agreement, because the stimulus projecting to the temporal side of the retina appears closer more frequently. Only in Condition $\mathrm{D}$, however, was the difference statistically significant $[t(10)=2.67, p<.05]$. The statistically significant violation noted in Experiment 1 can be described as an instance of a "tendency to perceive binocularly correlated areas in front of uncorrelated areas"' (Julesz, 1971, p. 260). The statistically significant agreement found in Condition D, however, does not fit this description. The area containing the cross and the area containing the disk in panel D (Figure 3) are also the uncorrelated areas, and together they appeared in front. Obviously, the tendency noted by Julesz or the prediction from Panum's rule cannot by itself account for these results.

The stimuli for Experiment 2 differ from what is generally considered to be the Wheatstone-Panum limiting case, and one can therefore ask whether they belong to the limiting case and whether we should have applied the rule to them. Although Panum (1858/1940) applied his rule to a stimulus that consisted of two concentric circles presented to one eye and one circle presented to the other eye, his stereogram that presents two lines to one eye and one line to the other eye is now almost synonymous with the limiting case. Wheatstone's (1838) stereogram (his Figure 23) also presented two lines to one eye and one 
line to the other, but in a different way. A thin vertical line intersecting a thick inclined line was presented to one eye, a thick vertical line was presented to the other eye, and the two thick lines were expected to fuse. The stereograms for Experiment 2 are analogous to Wheatstone's, in that they were designed to have one of the two stimuli potentially fuse with the single stimulus. Moreover, for the top portion of Wheatstone's stereogram the occlusion can operate, whereas for the bottom part it cannot. (See Ono \& Wade, 1985, for an analysis of Wheatstone's stereogram.) A possible problem with classifying stimuli such as those in Experiment 2 as instances of the limiting case is that the depth reported cannot easily be explained as usual, by saying that the depth is due to the single stimulus fusing with both stimuli (Hering, ${ }^{1}$ cited without reference by Ogle, 1962; Ogle, 1962; Westheimer, 1986; and see the General Discussion in the present paper). Perhaps the answer to the question of what constitutes the Wheatstone-Panum limiting case should be delayed until general acceptance or rejection of the occlusion hypothesis occurs.

\section{EXPERIMENT 3}

We tested the occlusion hypothesis further with a stimulus that was also analogous to Wheatstone's stereogram, but that differed from the stimulus of Experiment 2 in that the occluded and ambiguous conditions were presented simultaneously. We also compared the depth seen with that produced by a standard retinal disparity stimulus. For the experimental stimuli, three lines were presented to one eye and one to the other eye. When the middle image of the three was in binocular correspondence with the single one, the stimulus condition was analogous to that of Wheatstone's stereogram in that one portion of the stimulus could be interpreted as being due to occlusion but another portion could not. The image on the nasal side could be interpreted as originating from an object occluded by another object in the fixation plane, but there was no good interpretation for the origin of the image on the temporal side, if the middle one was interpreted as originating from the object in the fixation plane. (See Figure 4A.) This condition combines the two experimental conditions of Experiment 1 into one. When the most temporal image on one retina was in correspondence with the single one on the other retina, the stimulus was like that of the location-defined condition in Experiment 1, except that there were two images instead of one that could be interpreted as being occluded by the stimulus on the fixation plane. (See Figure 4B.) When the most nasal one was in correspondence with the single image, the stimulus was like that of the location-ambiguous condition, except that the origins of the two images instead of one were ambiguous. (See Figure 4C.) Accordingly, there were three experimental conditions in Experiment 3-namely, the modified Wheatstone, the location-defined, and the location-ambiguous conditions. For comparison, we presented two lines to each eye; one pair was in binocular correspondence, but the other pair had retinal disparity. We also presented three lines to each eye without disparity. By comparing the frequency of depth seen with these stereograms and that seen with the experimental stereograms, we wished to answer the question about how consistently the depth is seen in the Wheatstone-Panum limiting case.

\section{Method}

Stimuli and Apparatus. Using a Macintosh II computer instead of the Grinell Graphic System used in Experiments 1 and 2, we presented stereograms on a high-resolution monochrome monitor (M0400). Other parts of the apparatus, such as the arrangement of the sheets of Polaroid, prism, and lens were the same as in Experiments 1 and 2 . The apparatus created a virtual image in the median plane, $40 \mathrm{~cm}$ from the observer.

There were 11 stereograms: 2 stereograms for each of the three experimental conditions, and 5 stereograms for the comparison con-

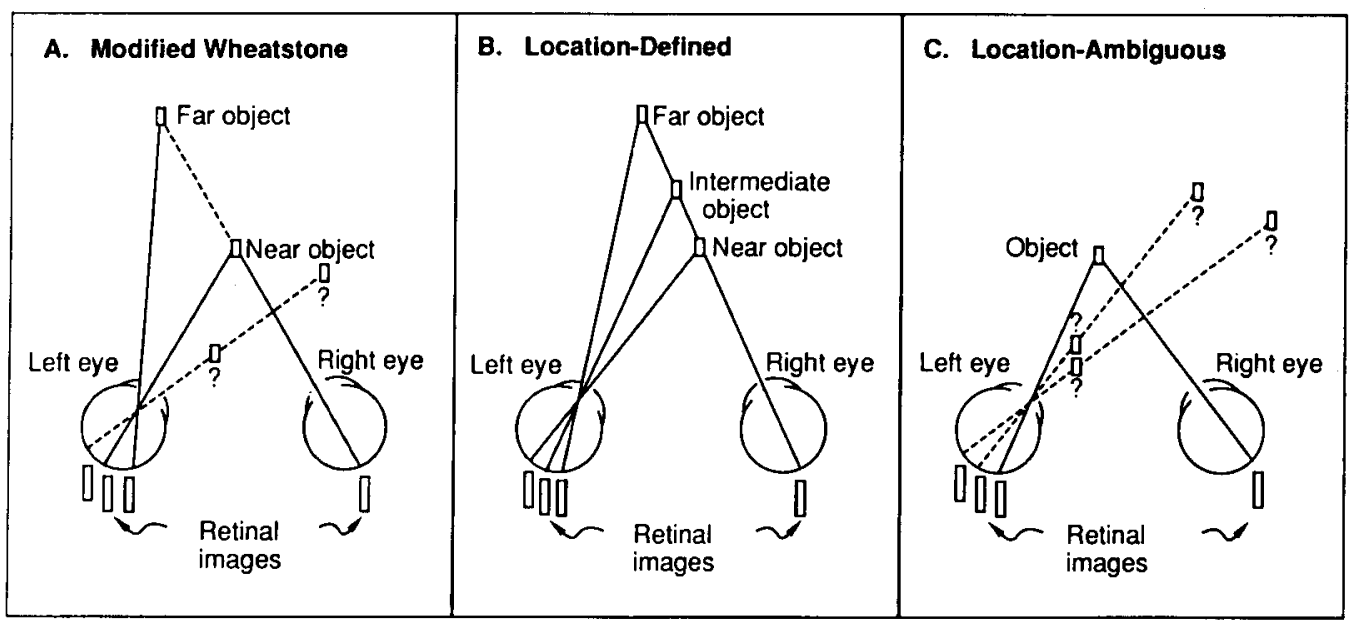

Figure 4. Three experimental conditions for Experiment 3: the modified Wheatstone condition (A), the locationdefined condition (B), and the location-ambiguous condition (C). 
dition. All the stereograms had the same "frame" to facilitate binocular fusion and to "lock" the convergence. The vertical lines of different thickness were haphazardly placed within the outer boundary and were the same for the two eyes. The nonuniform nature of the lines and their locations prevented the occurrence of the wallpaper illusion. The 11 stereograms differed in the number of test lines used and in the location of the nonius lines.

We will first describe the no-disparity stereogram from the comparison condition, because the differences among the 11 stereograms can best be described by referring to this one. It is shown in Figure 5. Three test lines were presented to each eye, and the separation between the adjacent lines subtended 17' of arc. The lines used in each stereogram were $59^{\prime}$ of arc high and $4^{\prime}$ of arc wide. The outer boundary of each field was $7^{\circ} 9^{\prime}$ of arc high and $6^{\circ} 27^{\prime}$ of arc wide. For the nonius stimulus, a thinner and longer line was presented to each eye. When the top one and the bottom one appeared to be collinear with the binocularly fused lines (in this case, the middle test lines), we assumed that the convergence was at the desired position.

For all three experimental conditions, stereograms were made by removing two of the three lines in one field of the no-disparity stereogram. The two stereograms for the modified Wheatstone condition were the same as the no-disparity stereogram, except that the left and the right lines were removed from one field. They presented the middle line of the three on the corresponding points of the single line. The stereograms for the two other experimental conditions were made by removing two adjacent lines from one field and by setting the nonius lines to be collinear with the binocular lines. The two stereograms for the location-defined condition presented the most temporal image of the three lines on one retina in correspondence with the single one on the other retina. The two stereograms for the location-ambiguous condition presented the most nasal image of the three lines in correspondence with the single one. There were two stereograms for each condition, because the two lines could be removed from either field shown in Figure 5.

For the four other stereograms in the comparison condition, one line was removed from each field shown in Figure 5 . The middle one of one field and the extreme left or right one from the other field were removed. For the stereogram in which the lines on the extreme left or right remained in both fields, nonius lines were placed above and below them. These stereograms presented one pair of lines on corresponding points and the other pair with retinal disparity of $17^{\prime}$ of arc. There were four stereograms, representing a $2 \times 2$ factorial: the extreme left or right lines in binocular correspondence, and the crossed or uncrossed retinal disparity produced by the two remaining lines. These four, along with the no-disparity stereogram shown in Figure 5, provided the five stereograms for the comparison condition.
Procedure. Unlike the viewing time in Experiments 1 and 2, which was limited to $100 \mathrm{msec}$, the viewing time in Experiment 3 was not limited. Each observer viewed a stereogram as long as he or she wished, and the trial ended when the observer finished reporting the relative position of the lines. This procedure is like that used by Ono and Wade (1985) in studying Wheatstone's original stereogram. The observers were asked to check the nonius alignment continuously during the stimulus presentation. For 13 observers, the alignment was maintained throughout the experiment, but for $1 \mathrm{ob}-$ server, it was not. For this observer, the experiment was restarted from the preparatory stage, during which the variable diopter prisms were readjusted. Each of the 11 stereograms was presented twice to each observer, and the order of presentation was random. Typically, the optical adjustment at the beginning took about $10 \mathrm{~min}$, and the 22 trials took about $20 \mathrm{~min}$.

Observers. Fourteen members of the university community-9 females and 5 males-participated. They ranged in age from 23 to 41 years, and they reported having normal or corrected-to-normal vision.

\section{Results and Discussion}

The results from the experimental conditions are reported first. We coded the perceived relative positions among the three perceived lines in each trial into the relative distance with respect to the fused line. The fused line was given the value of zero. Nonfused lines that appeared farther than, at the same distance as, or closer than the fused line were given a positive, zero, or negative value, respectively. The value of 1 or 2 was given to describe the depth relation between two nonfused lines. Thus, the possible range of values was -2 to +2 . The mean of the four values obtained for each observer in each experimental condition was computed. Figure $6 \mathrm{~A}$ summarizes the results from the modified Wheatstone condition, and Figure $6 \mathrm{~B}$ the two other conditions.

The modified Wheatstone condition provided results very similar to Ono and Wade's (1985) results obtained with Wheatstone's original stereogram. There was a great deal of variability within and between observers. Not many observers gave the same responses in the four trials, and the individual differences in the mean values can be seen in Figure 6A. Despite these variabilities, the central tendency is consistent with the occlusion hypothesis. The "occluded" nonfused stimulus (the nasal retinal im-

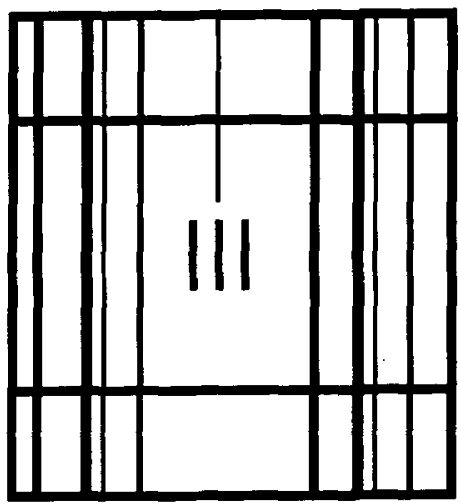

Figure 5. No-disparity stereogram from the comparison condition in Experiment 3. 
age) was seen as farther away. The mean depth value for the location-defined nonfused line was 0.61 and for the "ambiguous" nonfused line it was 0.14 . The difference was statistically significant $[t(13)=3.47, p<.01]$. Figure $6 \mathrm{~A}$ shows that 11 observers on the average saw the line as farther away, 2 observers reported no depth, and 1 saw it closer. The likelihood of this happening by chance is very low (sign test, $p<.01$ ), and the direction of the difference agrees with the prediction of the occlusion hypothesis. The hypothesis makes no prediction about the ambiguous nonfused line (the temporal retinal image). Figure 6A shows that 5 observers saw it as being farther, 5 saw no depth, and 4 saw it as being closer.

The location-defined and the location-ambiguous conditions together provided results consistent with those of the modified Wheatstone condition. A one-way repeated measures analysis of variance was performed on the data. The main effect was statistically significant $[F(3,39)=$ $8.70, p<.001]$. No observer saw the occluded nonfused lines as being closer in the location-defined condition (Figure $6 \mathrm{~B}$ ). There was 1 observer who saw the $17^{\prime}$ of arc line as being equally distant with the fused line, but there were no such observations for the $34^{\prime}$ of arc line. The likelihood of either of these distributions' occurring by chance is very low (sign test, $p<.01$ ). The retinal images that can be interpreted as originating from a line that is occluded are, on the average, seen as farther away than the fused line (0.70 and 0.89 in Figure 6B), but the difference is not statistically significant. The ambiguous retinal images that cannot be interpreted this way produced smaller averages, 0.23 and 0.26 . The post hoc analyses (Tukey test) showed that each mean in the location-defined condition was significantly higher than each mean in the location-ambiguous conditions $(p<.05$ for the smallest difference).

Figure 6 reveals a great deal of variability among and within observers. The among observers variability is indicated by the scatter of data points in each array and the within variability is indicated by many means not being equal to a whole number. Only 5 observers in the modified Wheatstone condition gave the same responses in four trials, only 2 in the location-defined condition, and only 3 in the location-ambiguous condition. In contrast with these variabilities, there was a nearly perfect consistency on the stereograms in the comparison condition. The stereogram with disparity produced correct depth as well. The fused line that produced uncrossed disparity was seen as farther by all the observers four times. The fused line that produced crossed disparity was seen as closer by 13 out of 14 observers four times. Moreover, the three fused lines with no disparity were seen twice by all the observers as having no depth.

To make sure that this high variability in the experimental conditions was not due to larger numbers of possible percepts with the experimental stereograms than with the disparity stereograms, we constructed another set of 12 disparity stereograms. The stereograms in this set had three lines appearing at different distances and at different vertical and horizontal directions. Although the lengths and widths of the lines were the same as those of the ex-

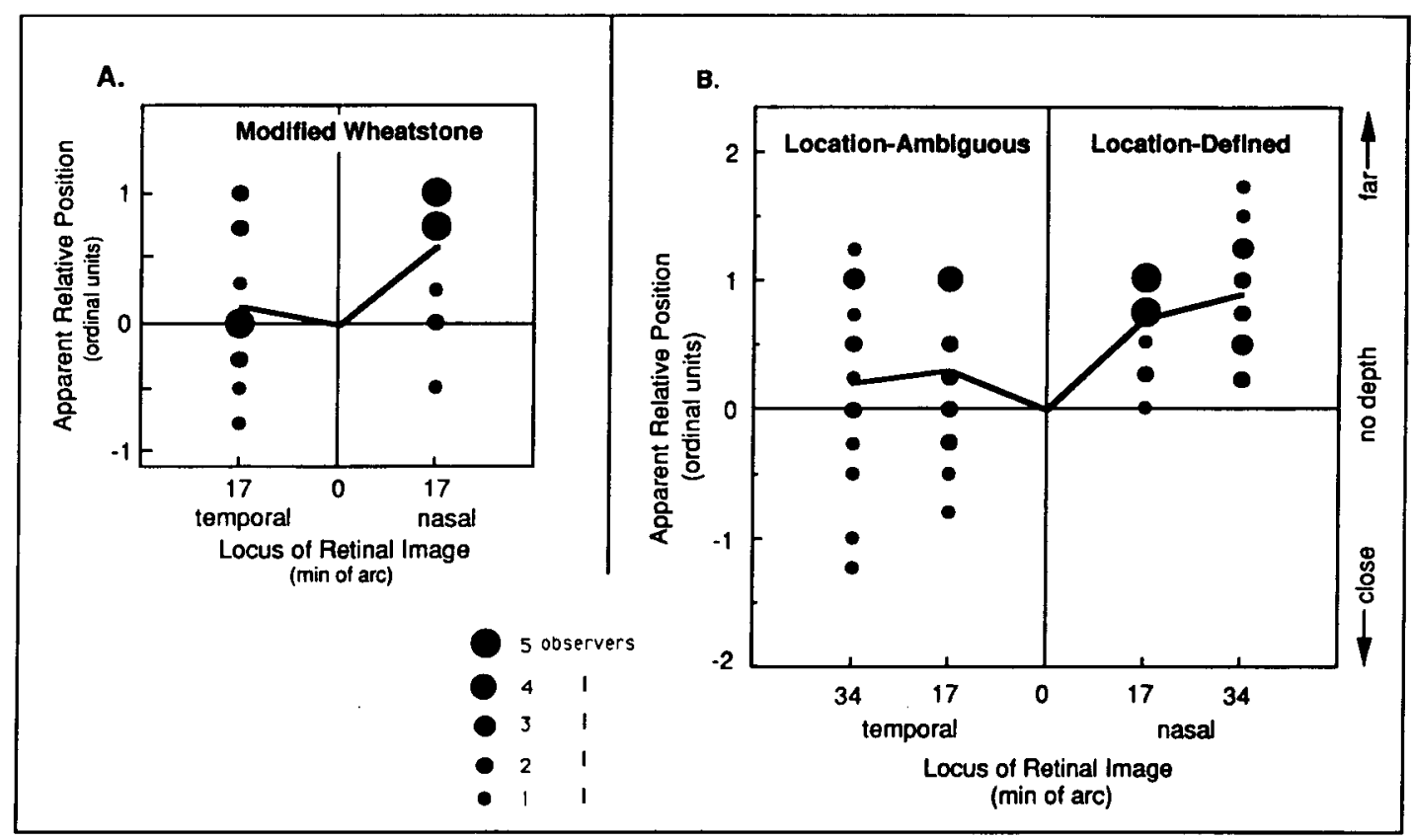

Figure 6. Apparent relative depth as a function of retinal locus in three experimental conditions: the modified Wheatstone condition $(A)$ and the location-ambiguous and location-defined conditions $(B)$. The data point represents the mean of apparent relative position for an observer. The size of the circle indicates the number of observers with the same mean. The line overlaid on the scatter graphs represents the group mean. 
perimental stereogram, the two pairs of lines were differently displaced up or down and leftward or rightward. The remaining pair were of the original height, had no disparity, and were accompanied by a nonius line. There were three subsets of stereograms: (1) with $8^{\prime}$ of arc of crossed and $8^{\prime}$ of arc of uncrossed disparity, (2) with $8^{\prime}$ and $17^{\prime}$ of arc of uncrossed disparities, and (3) with $8^{\prime}$ and $17^{\prime}$ arc of crossed disparities. The differences among the four stereograms in a subset consisted in whether the disparate pair appeared at the top or the bottom and on the left or the right. Five of the 14 observers returned to view these stereograms. All of them made correct responses in all trials. This supplementary result clearly indicates that the high variability found with the experimental stereograms was not due to the larger number of possible percepts.

The high variability in responses to the experimental stimuli was not due to a short viewing time in this experiment, since, unlike in Experiments 1 and 2, observers had unlimited viewing time. Moreover, it was not due to the artificial nature of the stimulus, because the disparity stereograms produced highly consistent responses. The high variability, we think, was due to occlusion's being a weak cue to depth. Because it is a weak cue, different tendencies, such as that of the uncorrelated area to appear farther than the fixation plane or of the monocular stimulus to appear equidistant with the fixation plane, began to play some role in determining apparent depth. This explanation not only accounts for the relatively high variabilities found in all three experiments for this study, but also for the variabilities across different studies going back to the inception of the stereoscope (see Ono \& Wade, 1985). An implication is that unlike with the depth seen with a retinal disparity, inferences should not be made about the binocularly defined occlusion from a small number of observers.

\section{GENERAL DISCUSSION}

The results of the three experiments support Ono and Wade's (1985) hypothesis about the perceived depth in the Wheatstone-Panum limiting case. The experimental conditions in which occlusion was more likely to operate as a cue for depth produced a higher percentage of perceived depth in accordance with Panum's empirical rule. These results and the recent reports that the visual system uses the binocularly defined occlusion cue (Nakayama \& Shimojo, 1990; Shimojo et al., 1988) make the occlusion hypothesis a viable one to explain depth seen in the Wheatstone-Panum limiting case. Given this conclusion, other hypotheses-namely the double fusion and the convergence error hypotheses-will now be addressed. A question is whether the occlusion hypothesis should be considered as a complementary or a competing one. A definitive answer cannot be given to this question because the hypotheses are not explicit enough for one to discriminate among them, but some comments are in order. ${ }^{2}$
The double fusion hypothesis (Hering, cited by Ogle, 1962; Ogle, 1962; Westheimer, 1986) is based on an assumption that both images on one retina fuse with the single image on the other retina. According to this hypothesis, depth is seen because one of the two fusions produces one disparity and the other fusion produces the other disparity. Two attractive features of this hypothesis are that it employs only the concepts used in stereopsis and that it explains the depth seen with Panum's stimulus, in which two concentric circles are presented to one eye and one circle to the other eye. However, whether it applies to our stimulus situations is not clear. We have assumed that the double fusion could not occur in Experiment 2 because of the differently shaped stimuli, but did it occur in Experiments 1 and 3 ? In this study, care was taken to place one of the retinal images in one eye so that it had binocular correspondence with the image of the single stimulus in the other eye. Did this procedure preclude double fusion and force a single fusion in Experiment 1? Can there be a triple fusion in Experiment 3? Whatever the answers to these questions, the point to note is that logically the process postulated in the double fusion hypothesis can be a complementary rather than a competing one. For example, if it is the case that for a double fusion to occur the values of the uncrossed and the crossed disparities must be approximately equal, then occlusion and double fusion can together contribute to the depth in accordance with Panum's rule, when the eye position is such that both uncrossed and crossed disparities are produced.

The convergence error hypothesis (Kaufman, 1976; Krol, 1982) is based on two assumptions: (1) one of the two images in one eye fuses with the single image in the other eye, and it appears in front of or behind the fixation plane, depending on the sign of the disparity produced by the convergence state; and (2) the nonfused stimulus appears on the fixation plane. These assumptions by themselves do not predict depth in accordance with Panum's rule; depth in accordance with the rule arises because of a tendency to underconverge (Kaufman, 1976) and a tendency to fuse the single image with the temporal image. (The second tendency is not made explicit in the hypothesis, but it is necessary for predicting the depth in accordance with the rule.) The simplicity of these assumptions makes this hypothesis attractive, but it makes no predictions for the three experiments in this study. If the nonius method is completely effective in producing no retinal disparity for the fused stimuli as intended, this hypothesis predicts no depth. According to this hypothesis, two perceived objects should appear in the fixation plane in all three experiments. How strongly the postulated process determines the depth perception when eye position is not controlled cannot be answered by this study. The point to note is that the process itself, like that postulated by the double fusion hypothesis, is not necessarily in competition with that postulated by the occlusion hypothesis.

What form the final explanation will take is difficult to say, but our study shows that occlusion is one of the 
factors in determining depth, albeit not as strong as retinal disparity. Moreover, our study shows that there are factors operating that are not considered in any of the three hypotheses, as indicated by the violation of Panum's rule noted in Experiment 1 and by the agreement in one of the contradictory-cue conditions in Experiment 2. These data cannot be explained by any of the three hypotheses, including the occlusion hypothesis. Despite its early discovery, there appears to be some way to go before we arrive at a complete understanding of the WheatstonePanum limiting case.

\section{REFERENCES}

ARDItI, A. (1986). Binocular vision. In K. R. Boff, L. Kaufman, \& J. P. Thomas (Eds.), Handbook of perception and human performance (Vol. 1, chap. 23). New York: Wiley.

BorING, E. G. (1933). The physical dimensions of consciousness. New York: Century.

BrewSTER, D. (1844). On the law of visible position in single and binocular vision and on the representation of solid figures by the union of dissimilar plane pictures on the retina. Transactions of the Rbyal Society of Edinburgh, 15, 349-368.

GeTtYS, C. F., Harker, G. S. (1967). Some observations and measurements of the Panum phenomenon. Perception \& Psychophysics, 2, 387-395

HerING, E. (1977). The theory of binocular vision (B. Bridgeman \& L. Stark, Eds. \& Trans.). New York: Plenum. (Original work published 1868)

Hering, E. (1942). Spatial sense and movement of the eye (C. A. Radde, Trans.). Baltimore: American Academy of Optometry. (Original work published 1879)

JuLEsz, B. (1971). Foundations of cyclopean perception. Chicago: University of Chicago Press.

Kaufman, L. (1974). Sight and mind: An introduction to visual perception. New York: Oxford University Press.

Kaufman, L. (1976). On stereopsis with double images. Psychologia, 19, 224-233.

Kaufman, L., Lane, B. (1979). Depth perception relative to convergence distance. Investigative Ophthalmology \& Visual Science, 18(Suppl.), 174.

KRoL, J. D. (1982). Perceptual ghosts in stereopsis: A ghostly problem in binocular vision. Amsterdam: Rodopsi.

LinschoteN, J. (1956). Strukturanalyse der binokularen Tiefenwahrnehmung [Structural analysis of binocular depth perception]. Groningen: Wolters.

MARR, D. (1982). Vision. San Francisco: W. H. Freeman.

Nakayama, K., \& Shimojo, J. (1990). Da Vinci stereopsis: Depth and subjective occluding contours from unpaired image points. Vision Research, 30, 1811-1825.

OGLE, K. N. (1962). The optical space sense. In H. Davson (Ed.), The eye (Vol. 4, pp. 374-375). New York: Academic Press.

ONO, H., WADE, N. (1985). Resolving discrepant results of the Wheatstone experiment. Psychological Research, 47, 135-142.

Panum, P. L. (1940). Physiological investigations concerning vision with two eyes (C. Hubscher, Trans.). Hanover, NH: Dartmouth Eye Institute. (Original work published 1858)

Shimojo, S., Silverman, G. H., Nakayama, K. (1988). An occlusion related mechanism of depth perception based on motion and interocular sequence. Nature, 333, 265-268.

Shimono, K., ONo, H. (1986). Occlusion as a cue for the depth in Wheatstone-Panum's limiting case. Investigative Ophthalmology \& Visual Science, 27(Suppl.), 345.

WAlker, H. M., Lev, J. (1953). Statistical inference. New York: Holt.

WESTHEIMER, G. (1976). Oculomotor control: The vergence system. In R. A. Monty \& J. W. Senders (Eds.), Eye movements and psychological processes (pp. 55-64). Hillsdale, NJ: Erlbaum.

Westheimer, G. (1986). Panum's phenomenon and the confluence of signals from the two eyes in stereoscopy. Proceedings of the Royal Society of London: Series B, 228, 289-305.

WheATSTONE, C. (1838). Contributions to the physiology of vision Part the first. On some remarkable, and hitherto unobserved, phenomena of binocular vision. Philosophical Transactions of the Royal Society, 128, 371-394.

\section{NOTES}

1. We have searched the two books by Hering (Hering, 1879/1942, $1868 / 1977$ ) that are translated into English, but we were unable to find his statements concerning double fusion.

2. For historical interest, Wheatstone's (1838) and Panum's (1858/ 1940) discussions of their stimuli may be briefly described. Wheatstone (1838) discussed his stereogram as a stimulus to test his idea that "similar pictures falling on corresponding points of the two retinae may appear double and in different places" (p. 384). Panum (1858/1940) discussed the observations that he made with the use of his stimulus in terms of the projection theory. For example, he stated that "the apparent locus of a contour combined binocularly is determined by the intersection points of the projection lines' (p. 130). (See Boring, 1933, for a discussion of the projection theory.) If the "intersection" is taken as a fusion, $\mathrm{Pa}$ num's interpretation can be construed as advocating the double fusion hypothesis. The projection lines of the two retinal images intersect with the projection line of the single retinal image, and thus there are two intersections.

(Manuscript received July 2, 1990; revision accepted for publication August 12, 1991.) 